\title{
Dental Mission for Children - Vietnam
}

Please send any ideas for feature articles for
consideration to:
Rowena Milan,
British Dental Journal,
The Macmillan Building,
4-6 Crinan Street,
London
N19XW
Email: r.milan@nature.com

This article by Noel Perkins and Lan Jones describes the ongoing work of the charity Dental Mission for Children, which provides dental care and dental health education in socioeconomically deprived areas of Vietnam.

\section{Vietnam}

Vietnam is a country of 83 million people situated in South East Asia, and is bordered by Laos, Cambodia and China to the West and North, with the Gulf of Tonkin and the South China Sea forming its eastern boundary. Hanoi is the capital of Vietnam and is located in the north of the country, but Ho Chi Minh City (also known as Saigon) is the largest, with over 6 million people, and is in the south. It is predominantly a mountainous country, with densely populated fertile areas, notably the Red River and Mekong Deltas in the north and south respectively. The Mekong Delta has an area of 40,000 square kilometres and is known as Vietnam's 'breadbasket', although 'rice basket' would be a more accurate term! Vietnam is the World's third largest exporter of rice, after Thailand and the USA.

\section{Dental Mission for Children}

Dental Mission for Children (DMC) is a non-profit humanitarian organisation which aims to promote volunteerism in the international dental community, as well as with all persons of vision and compassion who wish to be part of a fulfilling and much needed dental outreach program. It was founded by Dr Lan Jones in 1996, after many years of active involvement in volunteer dentistry in the USA and West Africa. Dr Jones is originally from Saigon in South Vietnam, but received her education and training in the USA.

Corresponding author: Dr Noel Perkins, Dental Surgeon, St. Helen's Dental Practice, Cockermouth, Cumbria, CA139N0.Email:noeljperkins@gmail.com.

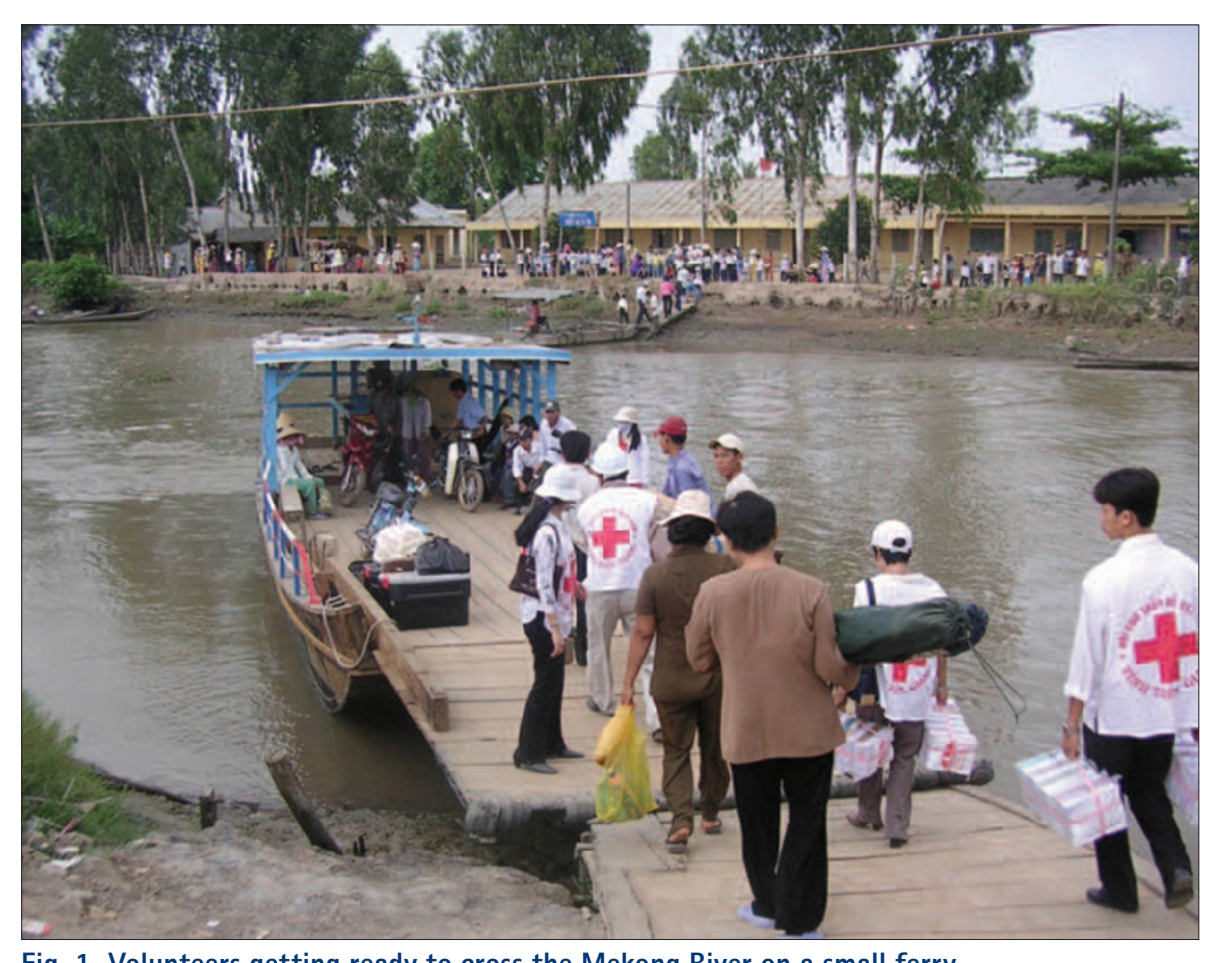

Fig. 1 Volunteers getting ready to cross the Mekong River on a small ferry

DMC sends teams of volunteers to serve local communities in socioeconomically deprived areas of Vietnam, providing primary dental care and dental health education. Over the last ten years, DMC has provided treatment for thousands of children in orphanages, urban centres for street children, elementary schools, special schools for physically disabled children, and remote tribal communities. There is an ongoing program of oral health education, which reaches out to tens of thousands of school children in rural areas of Vietnam throughout the school year. DMC works with local teachers in providing oral health education, as well as supplies of toothbrushes and toothpaste. DMC also assists disadvantaged children in rural areas to access medical and surgical care in city-based hospitals. In recent years financial support has been provided for many severely burned children, children with congenital or acquired facial deformity and children needing cardiac surgery. The local director of DMC in Vietnam, $\mathrm{Mr}$ Hung Dam, oversees these activities.

In addition to its work in Vietnam, DMC has established links with other humanitarian organisations - such as Teethsavers International - in order to support dental volunteers working with school children in Zambia and The Gambia. 


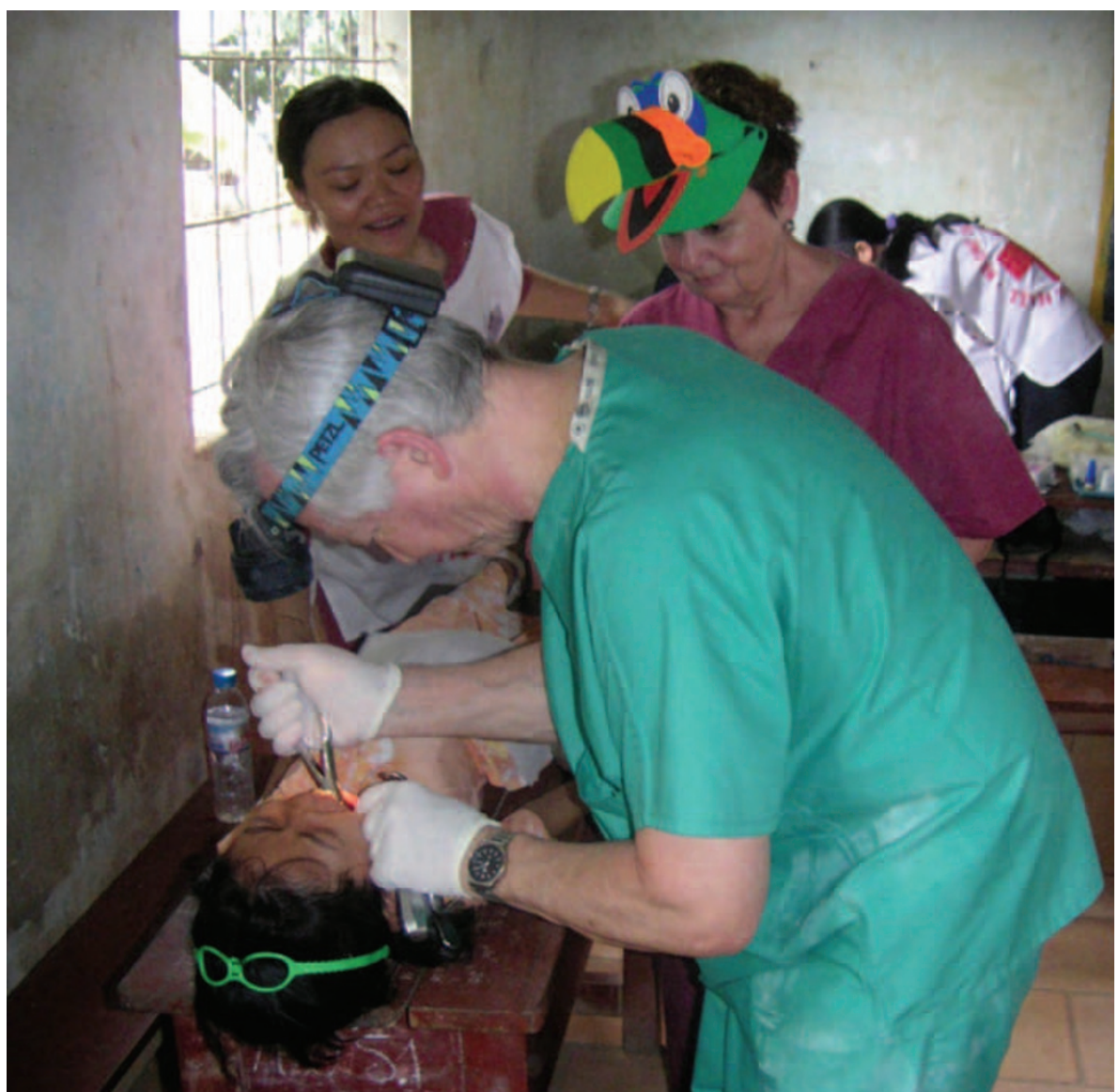

Fig. 2 Oral surgeon Dr Tom Love carrying out an extraction

\section{Mission trips}

The Mission trips to Vietnam usually last for a period of two weeks, and take place in March/April each year. Volunteers come from a variety of countries (eg USA, UK, Canada) and a broad range of backgrounds, including general dental practitioners, paediatric dentists, oral and maxillofacial surgeons, restorative dentists, dental students and non-dental support volunteers. DMC has developed strong links with the Vietnamese Red Cross and this has greatly facilitated the work of the charity in Vietnam. Volunteers from the Tien Giang Red Cross have been particularly helpful, and they continue to provide outstanding assistance with regard to translation and dental nursing support - which sometimes extends to mopping the perspiration off volunteers' brows and quenching their thirst with coconut water!

Volunteers are typically based in a small town in the Mekong Delta and then travel out daily to remote villages which have been identified by the Red Cross and DMC as having significant numbers of children in need of dental care. In some cases villages cannot be reached by road and small ferries must be used to cross branches of the Mekong River in order to reach the final destination (Fig. 1).

Field clinics are usually set up in one classroom of the local elementary school. A structured service delivery system is then implemented to ensure efficient workflow, as around 300-400 children are treated each day by the team. The children are first triaged by Dr Jones and their treatment needs prioritised. The need for dental treatment is always very high. The majority of children have never seen a dentist before and there are large amounts of untreated caries, as well as dental abscesses. The majority of treatment provided usually involves dental extractions (Fig. 2) and application of a topical fluoride solution (38\% silver diamine fluoride, Saforide ${ }^{\circledR}$, J. Morita Corporation, Japan) to deciduous and permanent teeth (Fig. 3). 0ccasionally excisional biopsies of benign

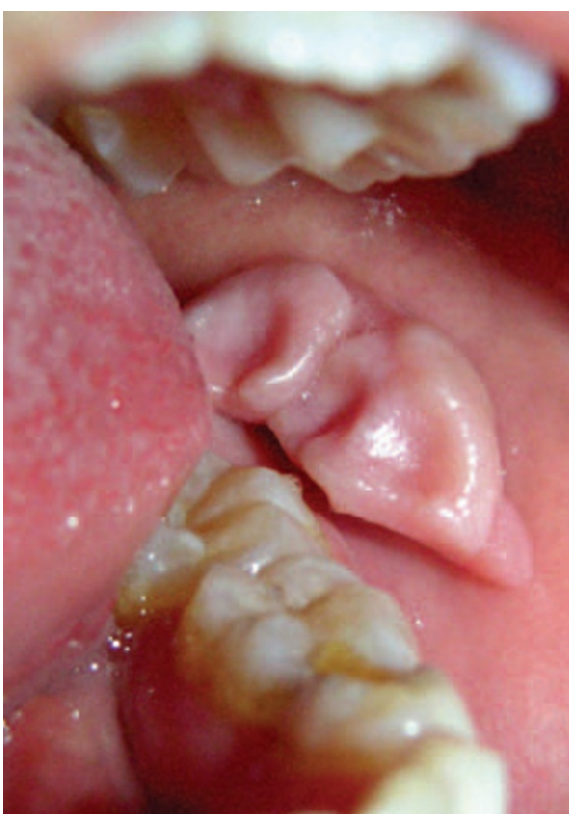

Fig. 4 Preoperative view of a large fibroepithelial polyp 
lesions, such as the large fibroepithelial polyp in Figure 4, are also carried out.

The level of cooperation of the children is excellent. They are always very respectful of their teachers and the members of the team, and listen attentively to the oral health education messages delivered by Dr Jones (Fig. 5). All children receive a Home Care Package consisting of a toothbrush, toothpaste, oral health guide in Vietnamese, exercise book and pencil. At the end of each afternoon treatment session, oral health education is also given to parents of preschool children.

The children and their parents are always very grateful for the treatment received. In some villages DMC donates wheelchairs to families of physically disabled children in order to help improve their quality of life. Witnessing the smiles on all the children's faces is an amazing and humbling experience (Fig. 6).

\section{Rest and relaxation}

Volunteers have the opportunity of visiting many unique sites in South Vietnam, including bustling cities like Saigon, My Tho and Vung Tau which are a great contrast to the quiet rural areas, where visits to pagodas, floating markets and botanical gardens are usually arranged. There is also the chance to explore tributaries of the Mekong River, which were largely controlled by the Viet Cong during the war but are now quiet waterways with peaceful glades overhung by tree ferns.

Due to the special friendship and trust developed by Dr Jones with the locals over many years, volunteers are also warmly welcomed into the homes of local families and have the opportunity to sample a fabulous range of Vietnamese cuisine and immerse themselves in Vietnamese culture.

\section{Volunteering}

Working with Dental Mission for Children can be a life-changing experience. Volunteers have the chance to build

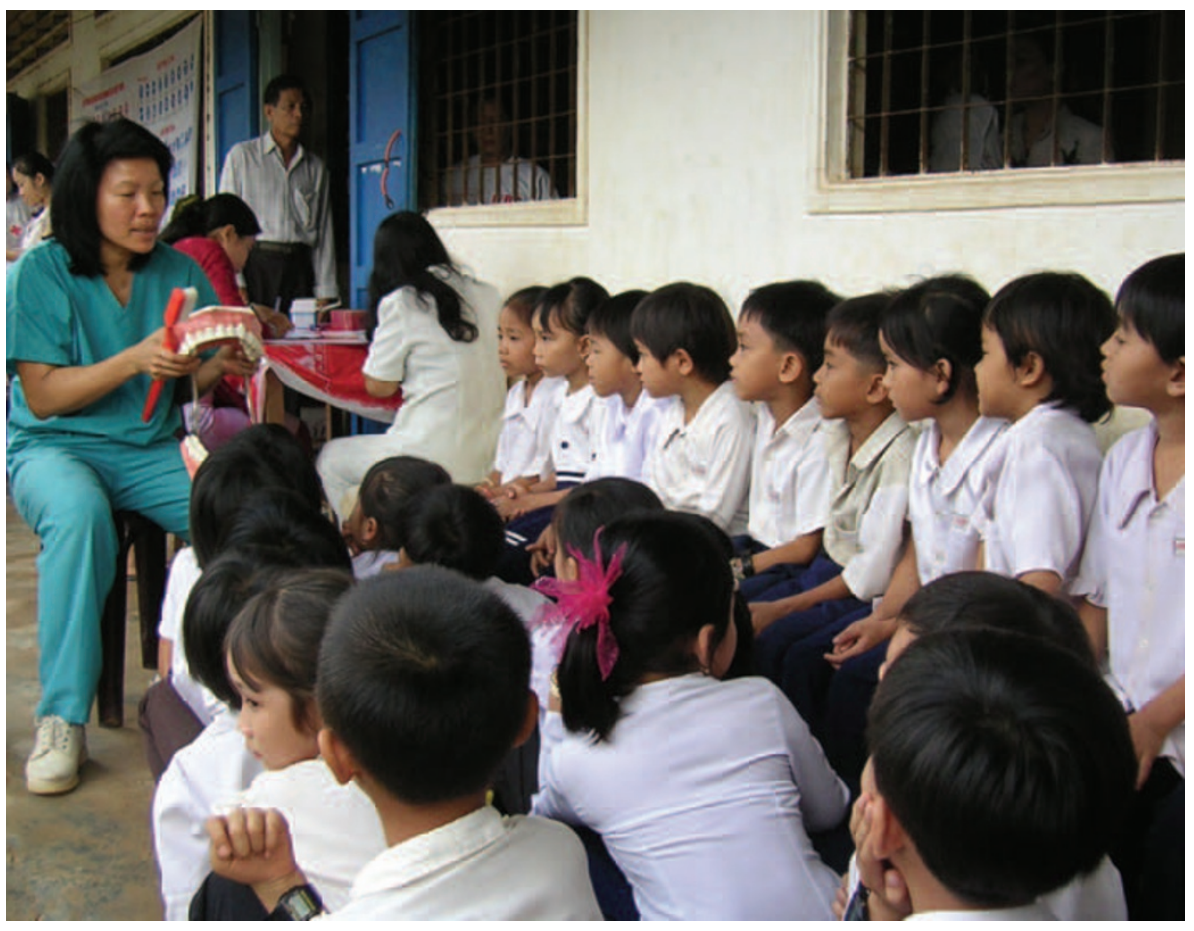

Fig. 5 Dr Jones giving oral hygiene instruction to an attentive audience of school children

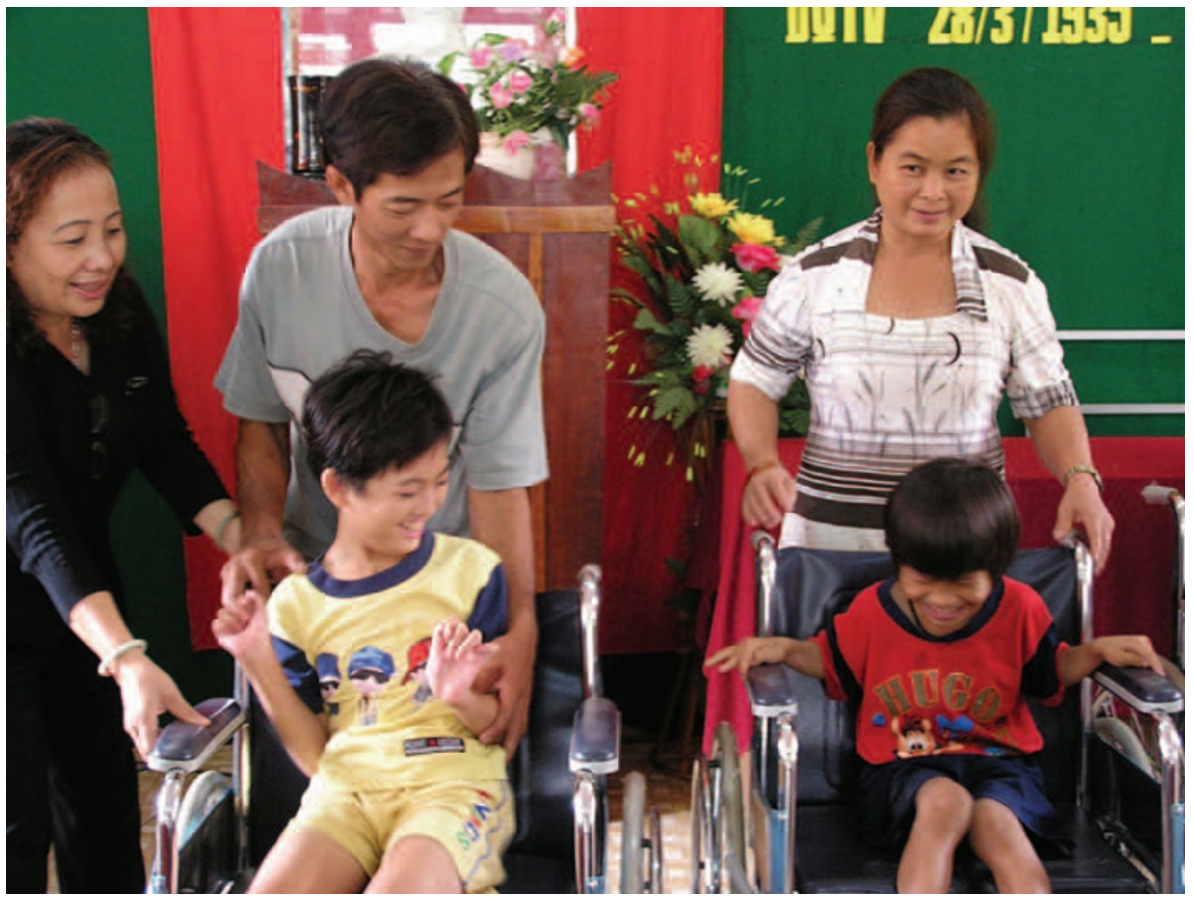

Fig. 6 Excitement of disabled children on receiving their first wheelchairs

lasting friendships with like-minded people from around the world and to touch the lives of thousands of children in need. Volunteers are able to develop a deep understanding and appreciation for Vietnam's history, culture, traditions, cuisine, and people.

If you would like to volunteer or would like more information, please contact $\mathrm{Dr}$ Lan Jones at lan@dds4kids.org and visit the website www.dds4kids.org.

DOI: 10.1038/sj.bdj.2009.50 